\title{
28 Research Square \\ Donor grafts from parents could help kids heal from ACL surgery
}

Emma L. Heath

Lucy J. Salmon

Robert Cooper

Evangelos Pappas

Justin P. Roe

MBBS

Leo A. Pinczewski

\section{Video Abstract}

Keywords: anterior cruciate ligament, reconstruction, living donor graft, hamstring tendon, autograft, allograft, reinjury, Tanner stage, puberty, graft survival, graft rupture, juvenile, adolescent, pediatric, contralateral ACL, survivorship, medium-term, American Journal of Sports Medicine, AJSM

Posted Date: September 20th, 2019

DOI: https://doi.org/10.21203/rs.2.15036/v1

License: (c) (i) This work is licensed under a Creative Commons Attribution 4.0 International License.

Read Full License 


\section{Abstract}

Parents can do a lot to help kids get back on their feet after ACL surgery, from making sure they get the rest and nutrition they need to ensuring crucial physical therapy appointments are kept. A new study in The American Journal of Sports Medicine suggests a more personal investment might also be important to recovery. Researchers report that parents who donate a hamstring tendon for use as a surgical graft could improve their kids' outcomes for years. Although hamstring tendon autografts are a common choice for ACL reconstruction, pediatric patients have unique characteristics that complicate this option. Taking a graft from a child still in development may cause persisting hamstring strength deficits and problems with walking and running. It's also difficult to obtain a graft that's large enough to prevent reinjury. These issues prompted researchers to take a closer look at how kids fare after ACL reconstruction using a graft donated from a parent. The team reviewed the five-year outcomes of 247 patients who underwent ACL reconstruction with a donor graft. The patients ranged in age from 8 to 17 years and represented all 5 Tanner stages - a measure of how far along a child is in puberty. Interestingly, it seems that puberty has a lot do with surgical success. Graft survival in less physically developed kids was 3 times that observed in more mature adolescents. Prepubertal children also had a much lower rate of reinjury. The reasons for this division in outcomes are varied. Compared to adolescents, juveniles are probably protected from reinjury in part because of increased parental supervision. Adolescents are likely to have more freedom to return to higher-level, more-aggressive sports than younger kids. Adolescents also have a greater body mass, which translates into higher impact on joints during play. And there may well be distinct cellular responses between the two groups, with younger children healing faster. Although more work is still needed to understand these observations, the results support that donor grafts from parents are a good option for ACL reconstruction in children who aren't yet skeletally mature. 